\title{
Robotik in der Knie- und Hüftendoprothetik
}

\author{
Peter Reiner Aldinger, Steffen Joachim Breusch, Hans Mau, Volker Ewerbeck
}

\section{Zusammenfassung}

Nach Einführung der computerassistierten Operationsverfahren in Orthopädie und Unfallchirurgie haben insbesondere im deutschsprachigen Raum Operationsroboter für die Hüftendoprothetik schnell Verbreitung gefunden. Die Möglichkeit der CT-basierten präoperativen Planung und deren exakte Umsetzung durch einen Operationsroboter haben bei Ärzten und Patienten eine Welle der Begeisterung über eine neue Dimension der Präzision ausgelöst. Jedoch sind insbeson- dere in den vergangenen 2 Jahren auch Zweifel über den tatsächlichen Patientennutzen durch die Robotersysteme aufgekommen, die in den Medien öffentlich und polarisierend diskutiert wurden. Nach mittlerweile 7-jährigem klinischen Einsatz in Deutschland und einer schnellen flächendeckenden Verbreitung dieser Präparationshilfen, nicht zuletzt aufgrund massiven Drucks von Patienten und Medien, sollen die Vor- und Nachteile der Operationsroboter in der Hüft- und Knieendoprothetik näher beleuchtet werden.

\section{Einleitung}

In der konventionellen Endoprothetik von Hüfte und Knie erfolgt die Planung der Implantation anhand von transparenten Planungsschablonen, die auf ein standardisiert angefertigtes Röntgenbild projiziert werden. Die Operation selbst erfolgt manuell mit Sägen, Raspeln und Fräsen, wobei Passgenauigkeit und Stabilität der Implantate zu großen Teilen von Erfahrung, Können und Einschätzung des Operateurs abhängen.

Die Präparation des Femurschaftes erfolgt bei der konventionellen Hüftendoprothetik mit Hilfe von Formraspeln in aufsteigender Größe. Durch den Raspelvorgang wird der spongiöse, weiche Knochen in der Markhöhle teils entfernt, teils komprimiert. Das geschaffene Knochenlager ist jedoch bei dieser Präparationsart relativ rauh und die Kontaktfläche zwischen Endoprothese und Knochen beträgt im Schnitt 30-40\% der Oberfläche.

OP-JOURNAL 2002; 17: 54-59

(c) Georg Thieme Verlag Stuttgart · New York
Kritisch ist bei der Implantation von zementfreien Endoprothesen die Passgenauigkeit, die bei einer 2-dimensionalen Planung aufgrund von Projektionsfehlern nur bedingt abgeschätzt werden kann.

Fehlerhaft einliegende Hüftendoprothesenschäfte und nicht achsgerecht implantierte Knieendoprothesen können das Langzeitergebnis negativ beeinflussen.

Bei der Knieendoprothetik besteht im Grundsatz die gleiche Problematik. Insbesondere die Position und Ausrichtung der Sägeschnitte, die für die Rekonstruktion der anatomischen Beinachse wichtig sind, sind in hohem Maß an die Erfahrung und Können des Operateurs gekoppelt, aber auch die akkurate Präparation der Knochenflächen ist für die exakte Passung der Implantate von Bedeutung.

\section{Hintergrund der Roboterentwicklung}

Ende der 80er Jahre klagten nach Implantation der ersten Generation von unzementierten Hüftendoprothesenschäften viele Patienten über postoperative Ober- schenkelschmerzen, und insgesamt wurden nur unbefriedigende mittelfristige Ergebnisse mit diesen Implantaten der frühen Entwicklungsgeneration mit hohen Raten an Frühlockerungen erreicht. Dafür wurde unter anderem eine unzu-

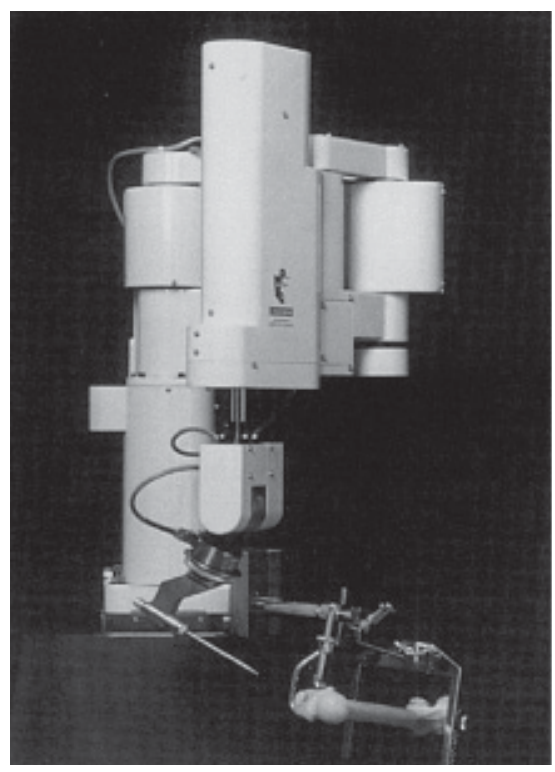

Abb. 1 Robodoc-Operationsroboter (ISS).

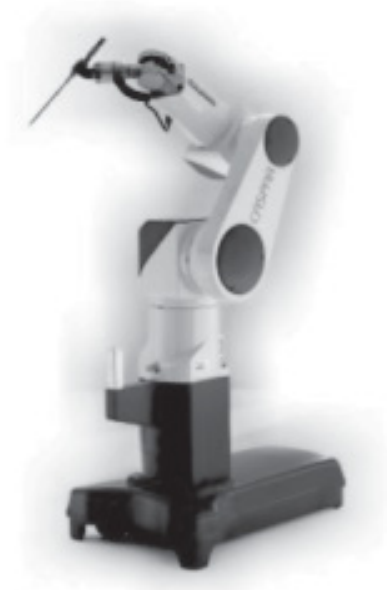

Abb. 2 CASPAR-Operationsroboter (URS-Ortho). 
reichende Passgenauigkeit der Implantate verantwortlich gemacht [4]. Um die operateurbedingten Ungenauigkeiten bei der Implantation zu minimieren und die Präzision der Präparation zu erhöhen, wurde Ende der 80er Jahre in den USA damit begonnen, Operationsroboter für die Fräsung des Markraumes zu konstruieren (Abb.1 u. 2).

Die Passgenauigkeit („Fit“) von zementfreien Schäften soll durch den Einsatz eines Operationsroboters verbessert werden [7].

\section{System}

Auf dem Markt sind zwei Robotersysteme vertreten: der Robodoc von ISS und der CASPAR von URS-Ortho (Abb.1 u.2). Im Grundsatz funktionieren die Robotersysteme nach dem gleichen Prinzip.

Das Robotersystem besteht aus einer Planungsstation mit einem Rechner, der die CT-Daten des Patienten verarbeitet und als dreidimensionales Bild rekonstruiert, einem zweiten Rechner im OP, der die Planung und die einzelnen Operationsschritte an den Operationsroboter überträgt und dem 5- oder 6-achsigen Roboter selbst, der die geplante Fräsung ausführt (Abb.3). An das Robotersystem ist eine Infrarotkamera gekoppelt, die Bewegungen des Patienten oder des OP-Tisches mit passiven Infrarotmarkern während des Eingriffs erfasst.

Die Form, Position und Orientierung der Fräsung wird präoperativ am Rechner geplant.

Während des Eingriffs wird der Knochen des Patienten über präoperativ implantierte Pinschrauben in allen räumlichen Ebenen erkannt und referenziert (Abb.4/ 9). Die Knochenpräparation erfolgt durch einen am Roboterarm installierten Hochgeschwindigkeitsfräskopf, der mit bis zu $75000 \mathrm{U} / \mathrm{min}$ rotiert und durch einen Wasserstrahl gekühlt wird (Abb.5).

\section{Pinimplantation und CT}

Um dem Roboter eine räumliche Orientierung am Patienten zu ermöglichen, ist bisher noch in der Regel eine Voroperation nötig. Für die Implantation einer Hüftendoprothese müssen dem Patienten in einem vorbereitenden operativen Eingriff - entweder zeitlich versetzt oder am gleichen Operationstag - zwei Markierungsschrauben („Pins“) im Bereich des Trochanter major und am dista-

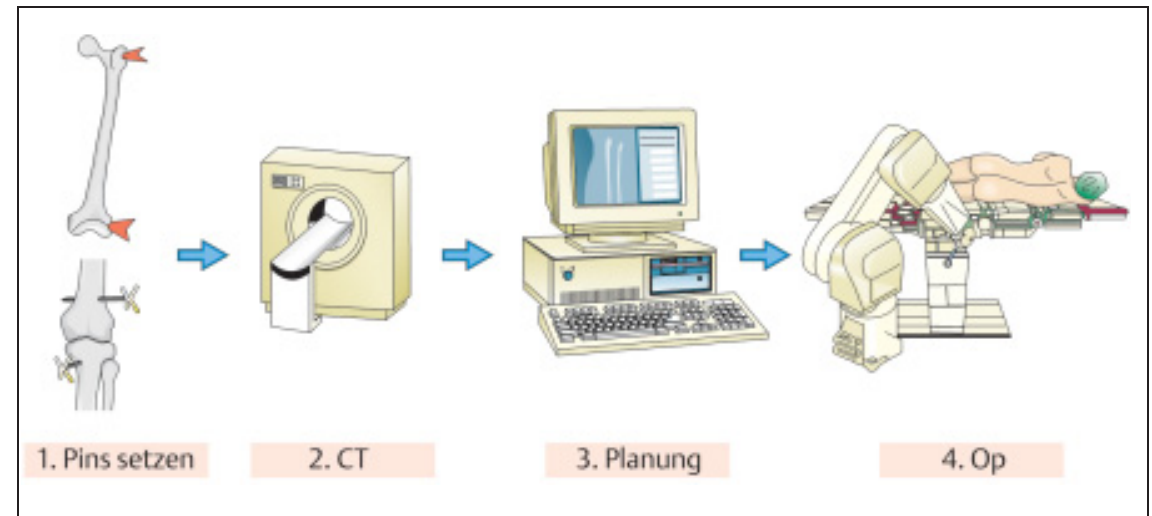

Abb.3 Ablaufschema einer Roboteroperation.

len Femur (oberhalb des Kniegelenks) implantiert werden (Abb.4). Für eine Knieendoprothese sind je ein Pin im distalen Femur und der proximalen Tibia erforderlich.

Voraussetzung für die Anwendung eines Operationsroboters ist ein Dünnschichtcomputertomogramm. Andere bildgebende Verfahren stehen für den Robotereinsatz momentan noch nicht zur Verfügung. Die CT-Schichten werden im Rechner zu einem dreidimensionalen Bild rekonstruiert und an einer Planungsstation weiterverarbeitet. Anhand der Markierungspins kann die Lage des Knochens intraoperativ berechnet werden.

\section{Planung}

Bei der Planung können mit Hilfe einer 3D-Rekonstruktion die Prothesenkomponenten frei in allen Ebenen geplant werden. Hierbei werden die entsprechende Prothesengröße, die Passung und die Gelenkgeometrie festgelegt (Abb.6u.7). Nach Abschluss der Planung werden die Daten gesichert und auf einem Datenträger zum Roboter transferiert.

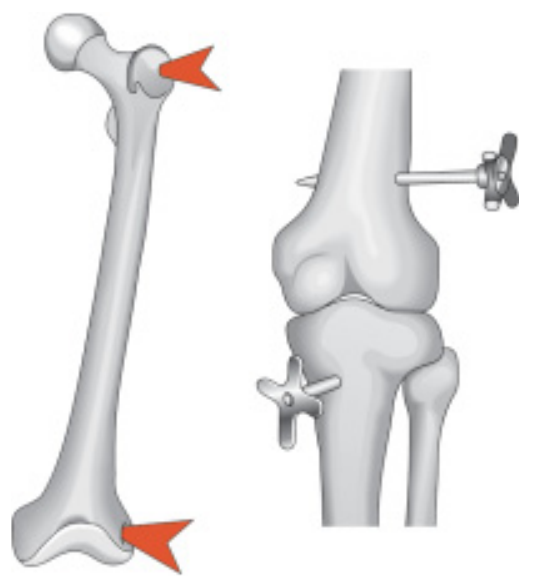

Abb. 4 Markierungs-Pins im Knochen zur Orientierung des Roboters.

\section{Vorbereitung}

Im OP wird etwa 30 min vor der Operation der Roboter positioniert und steril abgedeckt (Abb.8).
Abb. 5 Hochgeschwindigkeitsfräskopf zur Knochenbearbeitung.

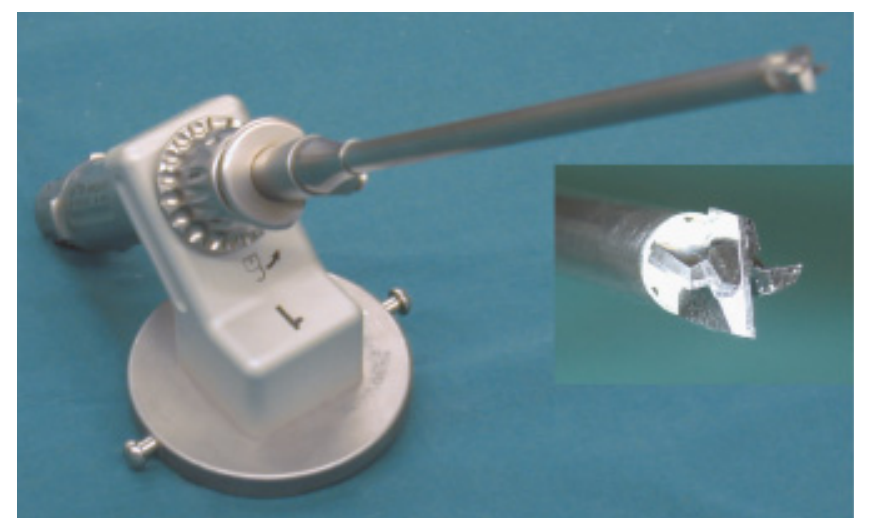




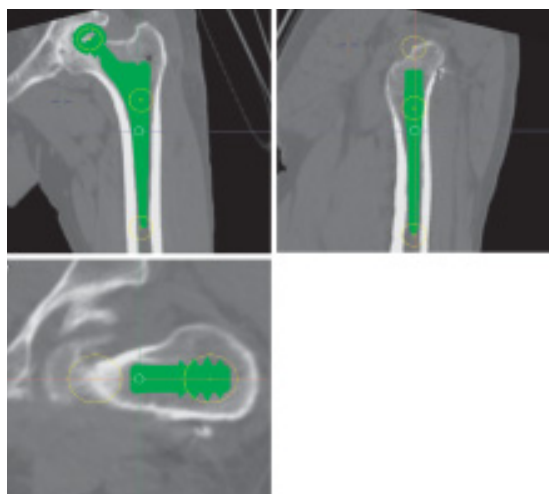

Abb. 6 3-dimensionale OP-Planung eines zementfreien Hüftschaftes.

\section{Lagerung}

Die Lagerung des Patienten ist für den Eingriff von entscheidender Bedeutung. Um bei der Hüftoperation den Weg des Fräsers von Weichteilen freizuhalten, sollte der Patient bei Operation in Rükkenlage so weit lateral wie möglich auf dem OP-Tisch gelagert werden.

Nach konventionellem Beginn der OP und Implantation der Pfanne erfolgt die Umlagerung in die Viererposition, wobei die Anspreizung und Abwinkelung des Beines mindestens $50-60^{\circ}$ betragen sollte, um die Arbeit des Roboters zu ermöglichen (Abb.10). Bei adipösen Patienten ist die Anwendung der Robotertechnik wesentlich schwieriger und der $\mathrm{Zu}$ gang wird deutlich größer.

\section{Fixierung des Beines}

Das betroffene Bein muss während des gesamten Robotereinsatzes fest in einem Beinhalter eingespannt sein, wobei das Femur fest in einer speziellen Haltezange fixiert wird. Hierfür muss die Muskulatur unterhalb des Trochanter minor abgelöst werden. Um Bewegungen, die eine korrekte Fräsung verhindern würden, zu vermeiden, erfolgt die Kontrolle der bewegungsfreien Fixierung mit einer Infrarotkamera im OP-Saal.

\section{Registrierung}

Während der Operation werden die Pins erneut dargestellt und durch einen am Roboterarm installierten und geeichten Taster registriert. So kann der Roboter die räumliche Position des Knochens mit dem Operationssitus vor der Fräsung abgleichen (Abb.9).

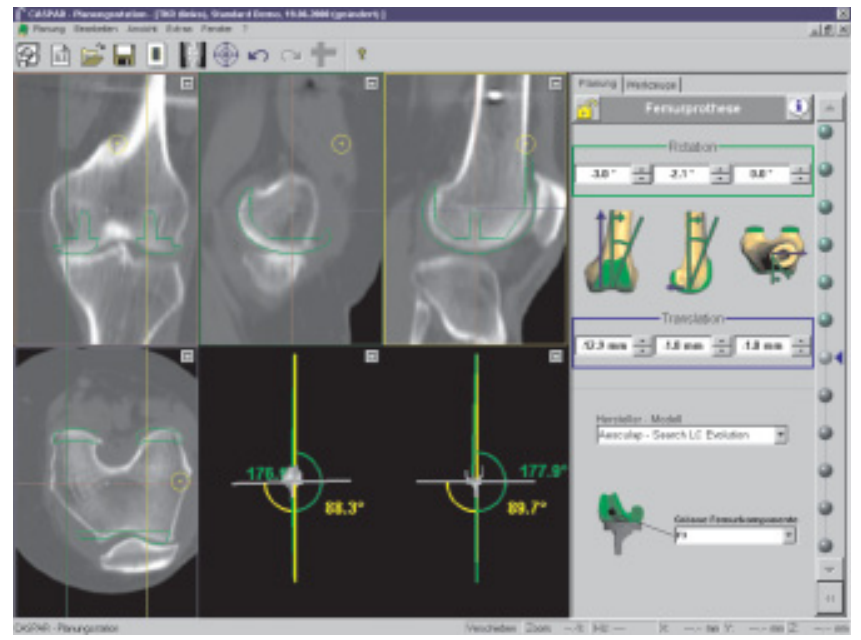

Abb. 7 3-dimensionale OP-Planung einer Oberflächenersatzprothese am Knie.

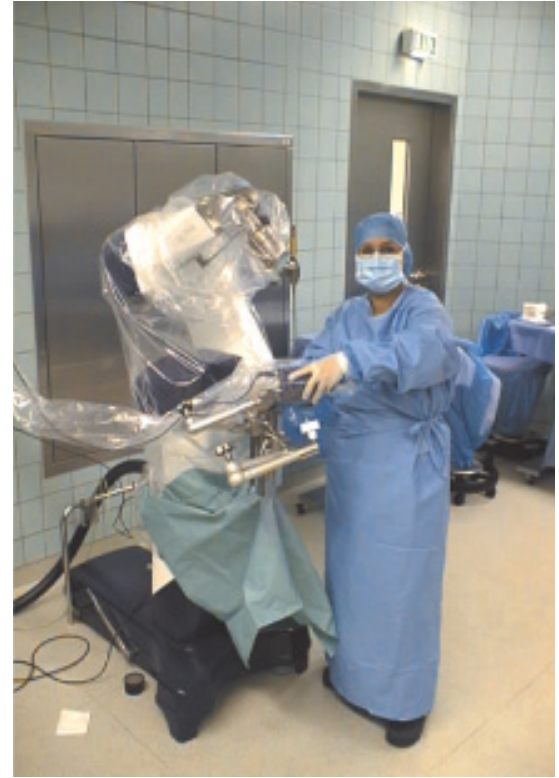

Abb. 8 Operationsroboter steril verpackt.

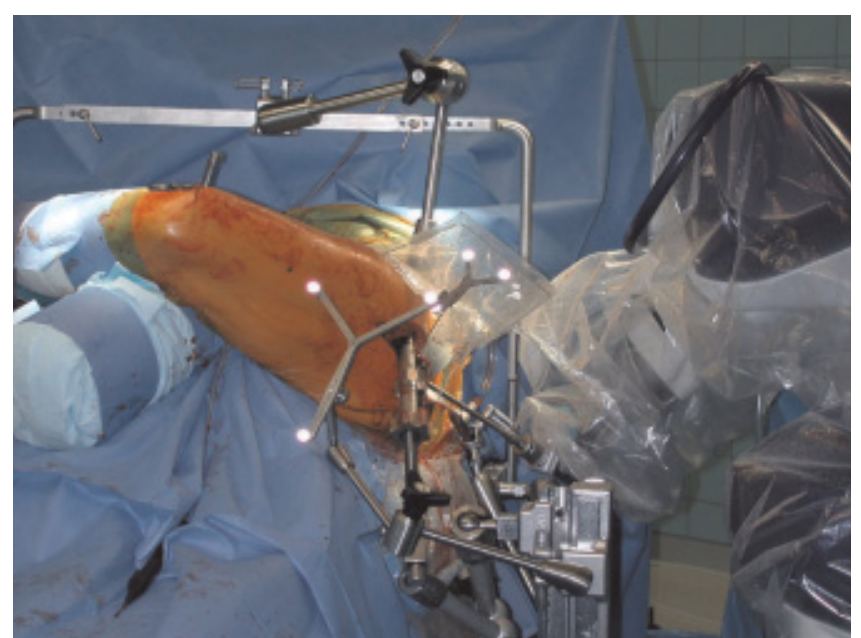

Abb. 10 OP-Situs bei Roboterfräsung eines Hüftschaftes. 


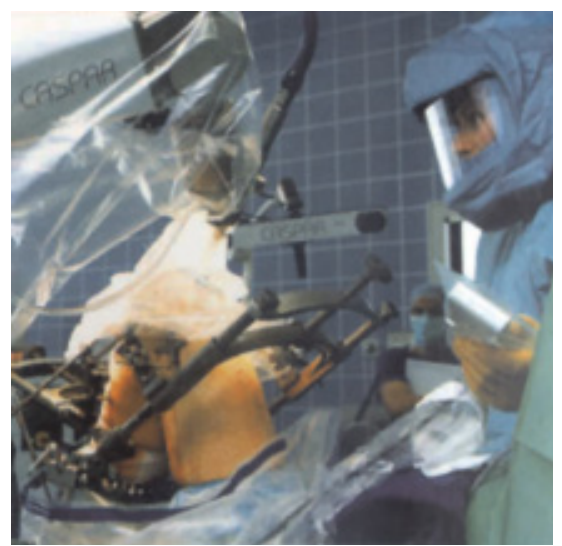

Abb.11 OP-Situs bei Roboterfräsung einer knieprothese.

\section{Fräsung}

Anstatt der Handraspeln, die mit einem Hammer nach Gefühl des Operateurs in den Femurschaft getrieben werden, übernimmt beim Robotereinsatz ein Hochgeschwindigkeitsfräskopf die Fräsarbeit (Abb. 5, 10,11). Der zeitliche Mehraufwand für Eichung und Fräsung beträgt bei routinierter Anwendung etwa 3045 Minuten.

\section{Vorteile der Roboterfräsung}

\section{Exakte Planung}

Die Roboterfräsung ermöglicht eine 3-dimensionale Auswahl von Prothesengröße und korrekter Position des Implantatlagers in allen Ebenen, wogegen bei der Handraspelung die Implantate lediglich 2-dimensional geplant werden können.

Die größere Planungsgenauigkeit gibt dem Chirurgen eine bessere Übersicht über die anatomischen Verhältnisse beim einzelnen Patienten.

\section{Exakte Positionierung}

Die Umsetzung dieser Planung erfolgt sehr exakt durch den Fräsarm des Roboters. Bei der Roboterfräsung wird die relativ unpräzise und operateurabhängige manuelle Raspeltechnik durch einen robotergeführten Fräskopf standardisiert.

Die Genauigkeit der Fräsung ist um ein 10faches höher als bei der Handraspelung.

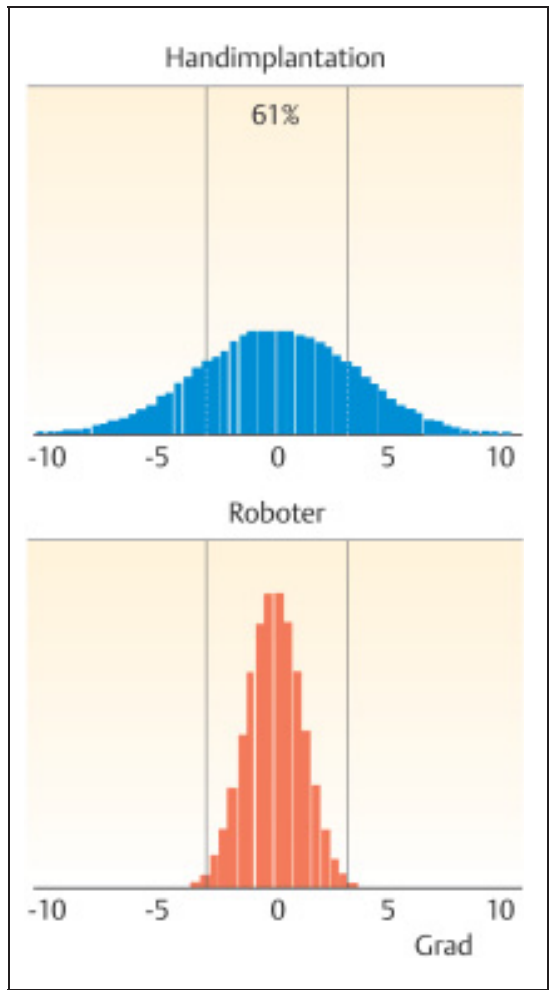

Abb.12 postoperative Beinachse bei Handund Roboterpräparation.

\section{Vergrößerter Knochenkontakt}

Es wird postuliert, dass über einen vergrößerten Kontaktbereich zwischen Implantat und Knochen die Primärstabilität, die für das Anwachsen des Implantates von entscheidender Bedeutung ist, durch die Roboterfräsung verbessert wird.

\section{Weniger Implantatfehllagen}

Bei Roboterimplantation können Implantatfehlpositionierungen im Vergleich zur Handimplantation verringert werden [1,9] (Abb. 12).

Bei schwierigen anatomischen Verhältnissen, wie nach Frakturen oder Umstellungsosteotomien, kann das System die Planung und Durchführung der Prothesenimplantation vereinfachen.

\section{Nachteile der Roboterfräsung (Tab. 1)}

\section{Pin-Implantation}

Ohne Zweifel liegt der größte Nachteil des Roboters momentan in der Notwendigkeit der vorbereitenden Pinimplantation.

Abgesehen vom zusätzlichen OP-Eingriff mit den jeweiligen OP-Risiken, klagen die Patienten häufig auch noch Monate bis Jahre danach über Knieschmerzen [5]. Diese Problematik würde im Zuge der Umstellung auf eine pinlose Anwendung entfallen. An einer pinlosen Registrierung für die Knieanwendung, wird zur Zeit noch gearbeitet. Voraussichtlich wird jedoch bis Mitte 2003 für Hüfte und Knie ein pinloses Verfahren zur Verfügung stehen.

\section{$C T$}

Durch das Computertomogramm wird der Patient einer nicht unerheblichen Strahlenbelastung ausgesetzt.

Tab. 1 Vergleich der Hand- und Roboterpräparation am Beispiel der Hüfte

\begin{tabular}{lll} 
& konventionelle Technik & Robotertechnik \\
\hline Vorbereitung & Röntgen & Röntgen \\
& - & Vor-OP (Pins) \\
& - & CT \\
Implantatbett-Bearbeitung & mechanisch mit Raspel, & Fräsung \\
& Säge & \\
Knochen-Implantatkontakt & ca. $30-40 \%$ & bis $90 \%$ \\
Spongiosa & kompaktiert/entfernt & entfernt \\
OP - Zeit & $60-90$ min & $120-150$ min \\
Blutverlust & normal (ca. $500 \mathrm{ml}$ ) & erhöht (ca. 1200 ml) \\
Nervenläsionen & bis $2 \%$ & bis $4 \%$ \\
TEP-Luxationen & unter 1,5\% & $3,20 \%$ \\
Fissuren & gelegentlich & selten \\
Schmerzen postoperativ: & Hüfte & Hüfte und Knie (Pin) \\
Zusatzkosten: & keine & Anschaffungspreis \\
& & Instandhaltungskosten \\
& & OP-Pauschale
\end{tabular}




\section{Planung}

Die Planung wird vom Roboter bedingungslos ausgeführt, auch bei Fehlplanungen fräst der Roboter programmgemäß.

\section{Fixierung}

Die starre Immobilisation des Beines über einen Zeitraum von etwa einer Stunde kann Thrombosen begünstigen, jedoch wurde bisher keine erhöhte Thromboserate publiziert [2].

\section{Lagerung}

Die weit laterale Lagerung lässt in der Regel den Einsatz eines C-Bogens nicht zu, somit ist die intraoperative Kontrolle der manuell implantierten Pfannen nicht möglich. Dieses Problem existiert nicht bei OP in Seitenlage. Die extreme Umlagerung des Beines kann zu Nervenschäden führen; deren Zahl liegt höher als in der von Hand operierten Vergleichsgruppe [1].

\section{Größerer Zugang}

Um die Arbeit des Roboters zu ermöglichen, muss der Zugang in vielen Fällen nach proximal erweitert werden. Außerdem müssen Teile der Abduktoren abgelöst werden, um den Eintrittspunkt des Fräskopfes in den Knochen darzustellen. Dies kann bei Hüftoperationen zu Irritationen der Abduktoren und nicht selten zu einem Trendelenburg-Hinken führen.

\section{Fräskopf}

Es ist schwierig, mit einem runden Fräskopf ein ideales Bett für eine eckige Prothese $\mathrm{zu}$ fräsen. Damit wird a priori das Postulat der Passgenauigkeit und verbesserten Stabilität infrage gestellt (Abb.5).

\section{Fehlende Pfannenplanung}

Bei der präoperativen Planung wird nur die femorale Seite des Implantates berücksichtigt, eine Pfannenplanung ist mit den Systemen momentan noch nicht möglich. Die optimale Pfannenpositionierung und das Bewegungsausmaß können somit noch nicht bestimmt werden. Ein mit dem Robotersystem verbundenes Pfannennavigationsmodul ist bei den Herstellern in Arbeit. Momentan liegt die Luxationsrate mit 3,2\% höher als bei der Handraspelung [1], wobei die genaue Ursache hierfür unklar bleibt.

\section{Hygiene}

Durch das rotierende mit einem Wasserstrahl gekühlte Fräsinstrument entsteht eine mit Partikeln kontaminierte Sprühnebelwolke zum Teil bis in den Bereich der Anästhäsie (cave: HIV, Hepatitis etc.).

Es muss daher auf ausreichenden Schutz der Mitarbeiter im OP (Schutzbrille etc.) geachtet werden [6].

Die Endoprothesenimplantation innerhalb eines Zeitraums von weniger als 10 Tagen nach der Pinimplantation ist aus hygienischer Sicht kritisch, da durch eine kontaminierte Wunde operiert werden muss.

\section{Kosten}

Zur Zeit kostet ein Operationsroboter etwa $500000 €$, der zugehörige Wartungsvertrag wird mit etwa $25000 € /$ Jahr berechnet. Pro Fall kommt auf den Anwender für eine Hüftanwendung eine Pauschale von $350 €$, bzw. für die Knieanwendung von $600 € \mathrm{zu}$, die mit der Fallpauschalenvergütung abgegolten werden muss. Des Weiteren müssen die Kosten für die Pinsetzung, das CT und der größere zeitliche Aufwand zu den anfallenden Kosten für die Operation addiert werden.

\section{Zeitbedarf}

Neben dem Zeitaufwand für Pinsetzung und $\mathrm{CT}$ müssen für den präoperativen Aufbau des Roboters mindestens $30 \mathrm{~min}$ eingeplant werden.

Die Operationszeit wird bei routiniertem Einsatz um etwa 30 - 50 Minuten verlängert, der Blutverlust ist höher als bei der konventionellen OP[1].

\section{Zusammenfassung}

Insgesamt ist festzuhalten, dass die Einführung der Roboter in die Endoprothetik einen Zugewinn an Planungsgenauigkeit und deren Umsetzung mit hoher Präzision gebracht hat.

Durch die robotergestützte Umsetzung der Implantatpositionierung kann das Risiko von Implantatfehllagen deutlich verringert werden.

Ob die erhöhte Präzision durch die Frästechnik bei der Bearbeitung der Knochenflächen eine Auswirkung auf das Einwachsverhalten und die Standzeit der Implantate hat, kann zum heutigen
Zeitpunkt nicht mit Sicherheit beurteilt werden. Ein wissenschaftlicher Beweis dafür konnte noch nicht erbracht werden [1].

In Laboruntersuchungen konnte nachgewiesen werden, dass die primäre Stabilität durch die Roboterfräsung in Abhängigkeit vom verwendeten Implantat nicht in jedem Fall verbessert werden kann, sondern sich bei manchen Implantaten im Vergleich zur Handimplantation sogar verringert [10].

Die kurzfristigen klinischen Ergebnisse bei Robotereinsatz sind mit denen der manuellen Implantation vergleichbar, auf die Rehabilitation hat die Roboterimplantation keinen positiven Einfluss.

Heute, über 10 Jahre nach Einführung des ersten Roboters, liegen die Langzeitergebnisse der zweiten Generation von zementfreien Hüftendoprothesenschäften, die konventionell implantiert wurden, vor.

Mit einer Überlebensrate von über 95\% nach über 10 Jahren dürften diese Ergebnisse auch durch die Roboterfräsung schwer zu überbieten sein [3].

Auch in der Knieendoprothetik liegen durch die Verbesserung der manuellen Operationstechniken die Überlebensraten im selben Bereich [8].

\section{Bewertung}

Bisher wurden seit 1994 etwa 10000 Hüft- und 1000 Knieendoprothesen mit Roboterhilfe implantiert.

Eine abschließende Bewertung der Robotersysteme ist zum jetzigen Zeitpunkt jedoch noch nicht möglich.

Als Alternative stehen für eine exakte Positionierung von Prothesenkomponenten heute schon verschiedene Navigationssysteme zur Verfügung, die zuverlässig und mit geringerem finanziellen Aufwand arbeiten. Die auf dem Markt befindlichen Robotersysteme befinden sich in einer ständigen Weiterentwicklung und haben ihr volles Potential noch nicht ausgeschöpft. Zur Zeit wird vorrangig neben der pinlosen Registrierung an einer Verringerung der Invasivität (kleinerer Zugang) und an der einer Einbeziehung der funktionellen Parameter (Bandspannung beim Knie, Planung des Bewegungsausmaßes bei der Hüfte) gearbeitet. Weiterhin sollte die Weiter- 
entwicklung der Roboter vorangetrieben werden, um in Anbetracht der hohen Kosten einen klinisch fassbaren Vorteil für den Patienten und den Chirurgen anbieten zu können. Mit der pinlosen Registrierung wird diese Forderung schon teilweise erfüllt.

\section{Literatur}

${ }^{1}$ Bargar WL, Bauer A, Börner M. Primary and revision total hip replacement using the Robodoc system. Clin Orthop 1998 Sep; 354 $82-91$

${ }^{2}$ Börner M, Bauer A, Lahmer A. Computerunterstützter Robotereinsatz in der Hüftendoprothetik. Unfallchirurg 1997 Aug; 100(8): $640-5$

${ }^{3}$ Delaunay C, Kapandji AI. Survival analysis of cementless grit-blasted titanium total hip arthroplasties. J Bone Joint Surg Br 2001 Apr; 83(3): 408-13

${ }^{4}$ Engh CA, Bobyn JD, Glassman AH. Porouscoated hip replacement. The factors governing bone ingrowth, stress shielding, and clinical results. J Bone Joint Surg Br 1987 Jan; 69(1): $45-55$

${ }^{5}$ Nogler M, Maurer H, Wimmer C, Gegenhuber C, Bach C, Krismer M. Knee pain caused by a fiducial marker in the medial femoral condyle: a clinical and anatomic study of 20 cases. Acta Orthop Scand 2001 Oct; 72(5): 477-80

${ }^{6}$ Nogler M, Wimmer C, Lass-Florl C, Mayr E, Trobos S, Gegenhuber C. Contamination risk of the surgical team through ROBODOC's high-speed cutter. Clin Orthop 2001 Jun; 387: 225-31

${ }^{7}$ Paul HA, Bargar WL, Mittlestadt B, Musits B, Taylor RH, Kazanzides P, Zuhars J, Williamson B, Hanson W. Development of a surgical robot for cementless hip arthroplasty. Clin Orthop 1992 Dec; 285: 57-66

${ }^{8}$ Ritter MA, Berend ME, Meding JB, Keating EM, Faris PM, Crites BM. Long-term followup of anatomic graduated components posterior cruciate-retaining total knee replacement. Clin Orthop 2001 Jul; 388: $51-7$

${ }^{9}$ Mai M, Siebert W, Heeckt PF. First Clinical experience with the new robotic surgery system CASPAR for total knee arthroplasty. (Submitted to J Arthroplasty)

${ }^{10}$ Thomsen M, Aldinger PR, Gortz W, Lukoschek M, Lahmer A, Honl M, Birke A, Nagerl H, Ewerbeck V. Die Bedeutung der Fräsbahngenerierung für die roboterassistierte Implantation von Hüftendoprothesenschäften. Vergleichende experimentelle Studie: Hand- vs. Roboterpräparation. Unfallchirurg 2001 Aug; 104(8): 692-9
Dr. med. Peter Reiner Aldinger Assistenzarzt

PD Dr. med. Steffen Joachim Breusch Oberarzt

\section{Dr. med. Hans Mau}

Oberarzt

Prof. Dr. med. Volker Ewerbeck

Ärztlicher Direktor

Stiftung Orthopädische

Universitätsklinik

Universität Heidelberg

Abteilung Orthopädie I

Schlierbacher Landstraße 200a

D-69118 Heidelberg 